\title{
Telomere length analysis in Cushing's syndrome
}

\author{
Anna Aulinas ${ }^{1}$, María-José Ramírez ${ }^{2}$, María-José Barahona ${ }^{3,4}$, Elena Valassi ${ }^{1,4}$, \\ Eugenia Resmini $^{1,4}$, Eugènia Mato ${ }^{1,5}$, Alicia Santos ${ }^{1,4}$, Iris Crespo ${ }^{1,4}$, Olga Bell ${ }^{1,5}$, \\ Jordi Surrallés ${ }^{2}$ and Susan M Webb ${ }^{1,4}$ \\ ${ }^{1}$ Endocrinology/Medicine Departments, Sant Pau Biomedical Research Institute, Hospital de Sant Pau, Universitat \\ Autònoma de Barcelona, Servei d'Endocrinologia, C/Sant Antoni Maria Claret, 167, 08025 Barcelona, Spain, \\ ${ }^{2}$ Department of Genetics and Microbiology, Center for Biomedical Network Research on Rare Diseases \\ (CIBERER Unit 745), Universitat Autònoma de Barcelona, Bellaterra, Barcelona, Spain, ${ }^{3}$ Endocrinology Department, \\ Hospital Universitari Mutua Terrassa, Terrassa, Barcelona, Spain, ${ }^{4}$ Center for Biomedical Network Research on Rare \\ Diseases (CIBERER Unit 747), ISCIII, Hospital de Sant Pau, Barcelona, Spain and ${ }^{5}$ Endocrinology Department, Center \\ for Biomedical Network Research on Bioenginnering, Biomaterials and Nanomedicine (CIBER-BBN), Hospital de \\ Sant Pau, Barcelona, Spain
}

Correspondence should be addressed to A Aulinas

Email

aaulinasm@gmail.com

\section{Abstract}

Introduction: Hypercortisolism in Cushing's syndrome (CS) is associated with increased morbidity and mortality.

Hypercortisolism also occurs in chronic depressive disorders and stress, where telomere length (TL) is shorter than in controls. We hypothesized that shortening of telomere might occur in CS and contribute to premature aging and morbidity.

Aim: To investigate TL in CS patients compared with controls.

Methods: Seventy-seven CS patients (14 males, 59 pituitary, 17 adrenal, and one ectopic; 21 with active disease) were compared with 77 gender-, age-, and smoking-matched controls. Fifteen CS were evaluated longitudinally, during active disease and after remission of hypercortisolism. Leukocyte TL was measured by telomere restriction fragment-Southern technique. Clinical markers were included in a multiple linear regression analysis to investigate potential predictors of TL. Results: Mean TL in CS patients and controls was similar (7667 vs 7483 bp, NS). After adjustment for age, in the longitudinal evaluation, TL was shorter in active disease than after remission (7273 vs 7870, $P<0.05)$. Age and dyslipidemia were negative predictors $(P<0.05)$, and total leukocyte count was a positive predictor for TL $(P<0.05)$. As expected, a negative correlation was found between TL and age (CS, $R=-0.400$ and controls, $R=-0.292 ; P<0.05)$. No correlation was found between circulating cortisol, duration of exposure to hypercortisolism or biochemical cure and TL.

Conclusion: Even though in the cross-sectional comparison of CS and controls no difference in TL was found, in the longitudinal evaluation, patients with active CS had shorter TL than after biochemical cure of hypercortisolism.

These preliminary results suggest that hypercortisolism might negatively impact telomere maintenance. Larger studies are needed to confirm these findings.

\section{Introduction}

Cushing's syndrome (CS), a rare disease due to excessive cortisol secretion, is associated with increased mortality and severe morbidity (increased cardiovascular risk and fatigability, osteopenia, neuropsychological alterations, and impaired health-related quality of life), not completely reversible after biochemical control (1).
European Journal of

Endocrinology

(2014) 171, 21-29
(C) 2014 European Society of Endocrinology Printed in Great Britain
The mechanisms by which these abnormalities do not recover completely appear to be complex and are not currently well understood. Hyperstimulation of the hypothalamic-pituitary-adrenal (HPA) axis also resulting in hypercortisolism may also occur in psychiatric diseases such as acute and chronic stress and post-traumatic stress 
disorder $(2,3)$. These situations are associated with poor health indexes, and telomere length (TL) has been found to be shorter than that in matched controls (4).

Telomeres are repetitive DNA sequences, located at the end of linear chromosomes, essential to maintain genomic stability. Without telomeres, genetic material could be lost after every cell division; thus, when telomeres are critically short, cell division stops and senescence and apoptosis are induced (5). To avoid telomere attrition and to maintain TL, germ-line cells and a few somatic cells produce an enzymatic complex called telomerase. Telomerase function can be regulated by genetic, epigenetic, environmental, and hormonal factors (5). These include mainly stress hormones such as cortisol, catecholamines, estrogens, and growth factors.

In this line, accelerated telomere shortening, higher levels of urinary catecholamines, and free urinary cortisol have been observed in situations with high perceived psychological stress (in sisters of patients with cancer, in acute mental stress) (6). In vitro studies have shown a 50\% reduction in telomerase activity in lymphocytes after exposure to high levels of hydrocortisone (7) and a rapid and dynamic loss of telomeric sequences after exposure of mice thymocytes to dexamethasone (8). Shorter leukocyte TL has been described to be associated with elevated cortisol responses and dysregulated patterns of daily cortisol secretion in women who are patient caregivers (9). Recently, a longitudinal study evaluating the association between coexisting changes in cortisol and telomerase activity in peripheral blood mononuclear cells has been published (10). The authors examined whether participation in mindfulness-based interventions and improvements in psychological distress and metabolic factors were associated with increases in telomerase activity. They observed that serum cortisol levels were negatively correlated with changes in telomerase activity, suggesting that changes in stress-related cortisol might be one of the signals regulating telomerase levels in humans.

This evidence led us to hypothesize that telomere shortening may be behind the increased morbidity and features of premature aging in patients with CS. Hypercortisolemia could contribute to premature aging by inducing accelerated telomere shortening, which in turn could be implied in the persistent morbidity and clinical consequences associated with CS, even years after biochemical remission. As TL is an indicator of chromosome stability, proliferative capacity, and cellular aging, measuring TL could contribute to the understanding of its clinical and biological significance. To the best of our knowledge, telomere dysfunction has not been evaluated in CS patients before.

The aim of this study was to investigate $\mathrm{TL}$ in patients diagnosed with CS compared with sex-, age-, and smokingmatched healthy controls and to evaluate whether normalization of the hypothalamic-pituitary-adrenal axis after treatment reverses possible abnormalities.

\section{Subjects and methods}

\section{Subjects}

In this case-control study, patients with endogenous CS followed in our institution since 1982 were eligible. Patients with adrenal carcinoma were excluded. Seventyseven CS patients and 77 controls, matched for gender, age, and smoking participated in the study. Fourteen were men $(18.2 \%)$ and 63 women (81.8\%). Mean age at the time of the study was $48.6 \pm 12.8$ years. Fifty-nine patients were of pituitary origin (76.6\%), 17 of adrenal origin (adrenal adenoma or bilateral macronodular hyperplasia), and in one patient the origin was unknown (ectopic ACTH secretion of unknown source). Twenty-one patients (27.3\%) had active disease at the time of the study and $56(72.7 \%)$ were cured; mean time of remission of hypercortisolism was $6.4 \pm 7.2$ years. Eight active CS patients (38\%) were treated with metyrapone, six (28.5\%) with ketoconazole, and three (14.2\%) with both drugs. Mean duration of endogenous hypercortisolism was 72 months (range 11-264). Duration of hypercortisolism was considered as the period between onset of symptoms (as referred by the patients) and remission of hypercortisolism (in patients in remission) or the time of current analysis (in active patients). The period between onset of symptoms and biochemical diagnosis of CS was 34 months (range 3-120). Twenty-two patients (28.6\%) had received pituitary radiotherapy and 71 (92.2\%) had undergone surgery. Fifty-three percent $(n=41)$ were cured after initial treatment and had no recurrence and $19.5 \%(n=15)$ were cured after further therapies for recurrent disease. Fifteen cured patients (19.5\%) were adrenal insufficient at the time of telomere analysis and required substitution therapy with hydrocortisone (mean dose $17.6 \pm 3.7 \mathrm{mg}$ and range $10-20)$. Nine patients (11.7\%) were GH deficient (four of which were replaced with recombinant human $\mathrm{GH}(\mathrm{rhGH})$ ); eight women (10.4\%) were gonadotropin deficient (all on estrogen/progesterone hormone replacement therapy); and 15 patients (19.4\%) were hypothyroid, ten due to thyroid-stimulating hormone (TSH) deficiency and five due to primary 
hypothyroidism (all on L-thyroxine ( $\left.\mathrm{L}_{-} \mathrm{T}_{4}\right)$ replacement). CS was considered in remission if either adrenal insufficiency was demonstrated (basal morning cortisol $<100 \mathrm{nmol} / \mathrm{l}(<4 \mu \mathrm{g} / \mathrm{dl})$ and/or undetectable 24-h free urinary cortisol) or morning cortisol suppression ( $<50 \mathrm{nmol} / \mathrm{l},<1.8 \mu \mathrm{g} / \mathrm{dl}$ ) after $1 \mathrm{mg}$ dexamethasone overnight was observed. Twenty-five patients (32\%) were on antihypertensive medication, $17(22 \%)$ on statin treatment for dyslipidemia, and 12 (16\%) were treated with calcium and vitamin D.

In a subgroup of 15 CS (all women) patients studied initially with active disease, a second analysis of TL was carried out once they were in remission. In this longitudinal study, three were of adrenal origin and 12 of pituitary origin. Mean age at the time of active disease was $43.5 \pm$ 12.1 years and at remission was $46.6 \pm 11.3$ years. The time elapsed between both analyses was $40.1 \pm 15.6$ months and mean time of remission was $28.5 \pm 14.1$ months. Three cured patients (20\%) were adrenal insufficient at the time of telomere analysis and required substitution therapy with hydrocortisone (mean dose $18.3 \pm 2.2 \mathrm{mg}$ and range $10-20)$; four patients (26.6\%) were hypothyroid, two due to TSH deficiency and two due to primary hypothyroidism (all on $\mathrm{L}_{-} \mathrm{T}_{4}$ replacement). None of the cured patients were GH-deficient; seven women (46.6\%) were postmenopausal at remission, but no gonadotropin deficiency was observed $(n=8)$.

Seventy-seven controls selected from the blood bank donor's database or from healthy volunteers recruited among hospital employees were matched for gender, age, and smoking status, three features known to affect TL. Namely, age is an important determinant of TL, typically decreasing with advancing age (11). Females usually present longer TL than males, because estrogens stimulate telomerase activity and protect DNA from reactive oxygen species-induced damage (12). Cigarette smoke constituents increase cumulative and systemic oxidative stress and inflammation, which induce increased white blood cell turnover, resulting in accelerated TL shortening (13). Medical history and physical examination excluded any who reported glucocorticoid exposure, severe and/or acute diseases, and severe psychiatric alterations (however, anxiety and mild depression were not exclusion criteria). Four controls $(5.7 \%)$ were on antihypertensive therapy, another four $(5.7 \%)$ were receiving statin treatment for dyslipidemia, and three (4.3\%) were treated with calcium and vitamin D.

Anthropometry (weight, height, BMI, and waist:hip ratio) was measured in patients and controls. Hypertension was defined as systolic blood pressure $>140 \mathrm{mmHg}$ or diastolic blood pressure $>90 \mathrm{mmHg}$ or the use of antihypertensive medications. Dyslipidemia was defined as total cholesterol (TC) $>220 \mathrm{mg} / \mathrm{dl}$, LDL > $130 \mathrm{mg} / \mathrm{dl}$, triglyceride levels $\geq 150 \mathrm{mg} / \mathrm{dl}$, or treatment with lipidlowering medication. Diabetes mellitus (DM) was confirmed with fasting glucose levels $>126 \mathrm{mg} / \mathrm{dl}$ in two consecutive determinations or 2 -h glucose after oral glucose tolerance test (OGTT) $>200 \mathrm{mg} / \mathrm{dl}$. Adult patients were considered osteopenic when $T$ score was $<-1$ and $>-2.5$ or osteoporotic when $T$ score was $<-2.5$ s.D.

All participants provided a blood sample for DNA extraction and gave their informed consent. The study was approved by the Hospital Ethics Committee.

\section{Methods}

Genomic DNA extraction from total leukocytes Genomic DNA extraction from total leukocytes was carried out using an adapted Proteinase $\mathrm{K}$ and Phenol protocol (14). Blood samples from the patients were collected in EDTA tubes to reduce DNA degradation. Genomic DNA was isolated from blood buffy coats. The buffy coat and white blood cell pellets were stored frozen at $-80{ }^{\circ} \mathrm{C}$ before processing. The white blood cell layers were harvested and digested with buffer containing $0.1 \mathrm{M}$ $\mathrm{MgCl}_{2}, 0.02 \mathrm{M}$ EDTA, $0.5 \%$ SDS, $0.01 \mathrm{M}$ Tris, pH 8.0, and $1 \mathrm{mg} / \mathrm{ml}$ of proteinase $\mathrm{K}$ at $37^{\circ} \mathrm{C}$ overnight. The lysates were homogenized by passing through a blunt 20-gauge needle $\left(0.9 \mathrm{~mm}\right.$ diameter) at $4{ }^{\circ} \mathrm{C}$ temperature and DNA was purified by phenol:chloroform:isoamilic alcohol (25:24:1) extraction, and ethanol precipitation. Finally, genomic DNA was dissolved in Tris-EDTA buffer and quantified by spectrophotometric analysis. The quality of genomic DNA was checked for high molecular weight by $1 \%$ agarose gel electrophoresis.

TL measurements $>$ TL measurements were carried out by the telomere restriction fragment assay (TRF) using the Telo TAGGG Telomere Length Assay Kit (Roche 12209136001). Briefly, $1 \mu \mathrm{g}$ of DNA was digested with 20 units of Rsal and Hinfl for $2 \mathrm{~h}$ at $37^{\circ} \mathrm{C}$. Samples were loaded on a $0.5 \%$ Seakem Gold Agarose gel and were run for $21 \mathrm{~h}$ at $35 \mathrm{~V}$. The gels were treated with $\mathrm{HCl}$, denaturalized and neutralized, and then transferred to a nylon membrane by capillarity for $12-18 \mathrm{~h}$. After fixation with u.v., hybridization was carried out with a DIG-labeled telomeric probe $\left(3 \mathrm{~h}\right.$ at $\left.42{ }^{\circ} \mathrm{C}\right)$. Finally, restriction washes, incubation with anti-DIG-AP antibody, and detection by chemiluminescence were carried out. Images were analysed with the program Quantity One. TRF mean was 
calculated using the formula: TRF mean $=\Sigma \mathrm{OD} i$ / $\Sigma(\mathrm{OD} i / L i)$, where $\mathrm{OD} i$ is the chemiluminescent signal and $L i$ is the length of the TRF fragment at position $i$ (15). A control sample, $2 \mu \mathrm{g}$ digested DNA derived from a single batch of HeLa cells, was run on each gel to minimize interassay variation. The mean TL for HeLa cells was $4113 \mathrm{bp}$, with a s.D. of $\pm 210 \mathrm{bp}$, which is in the acceptable range of accuracy of the Southern blot technique. The accuracy of southern blot technique is up to $\pm 300 \mathrm{bp}(16)$.

Biochemical, hormone, and bone analyses $~$ Routine serum determinations were carried out by standard automated laboratory methods: fasting glucose, TC, HDL and LDL cholesterol and triglyceride levels. Blood counts were made using automated cell counters. Twenty-four hours urinary free cortisol was measured with a commercial RIA with prior extraction with an organic solvent. Plasma ACTH, serum cortisol, and insulin-like growth factor 1 (IGF1) levels were measured using a commercial chemiluminescent immunometric assay. Lumbar spine and whole-body bone mineral density and bone mineral content were measured by DXA scanning (Delphi QDR 4500; Hologic); the mean precision error (coefficient of variation) was $1 \%$.

\section{Statistical analysis}

Statistical analyses were carried out using the SPSS 19.0 Statistical Package for Windows (SPSS, Inc.). Initially a descriptive analysis of all variables was carried out in order to verify correct introduction of data in the database. Quantitative data are expressed as mean and s.D. (Gaussian distribution) or as median and range (non-Gaussian distribution), and categorical data are expressed as percentages. Data distribution was analyzed by the Kolmogorov-Smirnov test. TL variable was normally distributed. Logarithmic transformations were carried out where necessary to normalize the distribution of a particular measure. Comparison between two groups was made using Student's t-test (Gaussian distribution) or Mann-Whitney's $U$ (non-Gaussian distribution)-test. A $\chi^{2}$ test was performed for categorical variables. Fisher's exact test was performed when appropriate. Pearson's correlation coefficient was used to estimate linear association between two quantitative variables. Analysis of covariance was performed to evaluate TL after adjustment for age and for total leukocyte count (as covariates). Multiple linear regression analysis including age, gender, BMI, type 2 DM, dyslipidemia, hypertension, psychiatric history, duration of hypercortisolism, current hypercortisolism, total leukocytes, and 24-h urinary free cortisol as potential predictive factors for TL (as dependent variable) was performed. $P$ values $<0.05$ were considered significant.

\section{Results}

\section{Comparison between CS and matched controls}

Main baseline characteristics of CS patients and controls are summarized in Table 1 . CS patients had more hypertension, diabetes, dyslipidemia, and osteoporosis than their matched controls $(P<0.05)$. Mean TL values in $\mathrm{CS}$ and controls are summarized in Fig. 1. No differences were observed between males and females (7732 \pm 1242 vs $7540 \pm 1361$ bp respectively). TL did not differ between CS and controls (7667 \pm 1260 vs $7483 \pm 1214$ respectively, NS). TL did not differ between active CS, cured CS (with or without secondary adrenal insufficiency) and their matched controls (Fig. 1).

As expected, a negative linear correlation between age and TL in the whole sample was observed $(R=-0.341$, $P<0.001)$. When both groups were evaluated separately, this negative correlation was maintained in CS patients $(R=-0.400, P<0.001)$ and in controls $(R=-0.292$, $P<0.01$ ) (Fig. 2). A positive correlation was found between IGF1 and TL in CS patients $(R=0.331, P<0.05)$, but was not correlated with the presence or absence of $\mathrm{GH}$ deficiency or rhGH replacement therapy. No differences

Table 1 Baseline characteristics of patients with Cushing's syndrome (CS) and controls. Data are presented as percentage and mean \pm s.D.

\begin{tabular}{|c|c|c|c|}
\hline & CS $(n=77)$ & $\begin{array}{c}\text { Controls } \\
(n=77)\end{array}$ & $\boldsymbol{P}$ \\
\hline Age (years) & $48.6 \pm 12.8$ & $48.4 \pm 12.6$ & NS \\
\hline Smokers (\%) & 24.7 & $1 \overline{9} .4$ & NS \\
\hline $\begin{array}{l}\text { Alcohol } \\
\text { consumption (\%) }\end{array}$ & 26 & 27.3 & NS \\
\hline $\begin{array}{l}\text { Diabetes mellitus } \\
\quad \text { (type 2) }(\%)\end{array}$ & 14.3 & 1.4 & $<0.05$ \\
\hline $\begin{array}{l}\text { Arterial } \\
\quad \text { hypertension (\%) }\end{array}$ & 57.1 & 12.9 & $<0.001$ \\
\hline Dyslipidemia (\%) & 45.5 & 20.0 & $<0.05$ \\
\hline Osteoporosis (\%) & 29.9 & 2.9 & $<0.001$ \\
\hline Psychiatric history (\%) & 37.7 & 11.4 & $<0.001$ \\
\hline BMI $\left(\mathrm{kg} / \mathrm{m}^{2}\right)$ & $28 \pm 5.6$ & $26.4 \pm 4.9$ & $<0.05$ \\
\hline Waist:hip ratio & $0.92 \pm 0.07$ & $0.85 \pm 0.07$ & $<0.05$ \\
\hline $\begin{array}{l}\text { 24-h Urinary free } \\
\text { cortisol (nmol/24 h) }\end{array}$ & $266 \pm 180$ & $132 \pm 59$ & $<0.001$ \\
\hline $\begin{array}{l}\text { Morning serum } \\
\text { cortisol }(\mathrm{nmol} / \mathrm{l})\end{array}$ & $450 \pm 259$ & $375 \pm 120$ & $<0.05$ \\
\hline Leukocytes (×109/l) & $7.3 \pm 2.3$ & $5.8 \pm 1.7$ & $<0.05$ \\
\hline Neutrophils $\left(\times 10^{9} / \mathrm{l}\right)$ & $4.4 \pm 2.0$ & $3.5 \pm 1.2$ & $<0.05$ \\
\hline Lymphocytes $\left(\times 10^{9} / \mathrm{I}\right)$ & $2.1 \pm 0.8$ & $1.9 \pm 0.4$ & NS \\
\hline
\end{tabular}




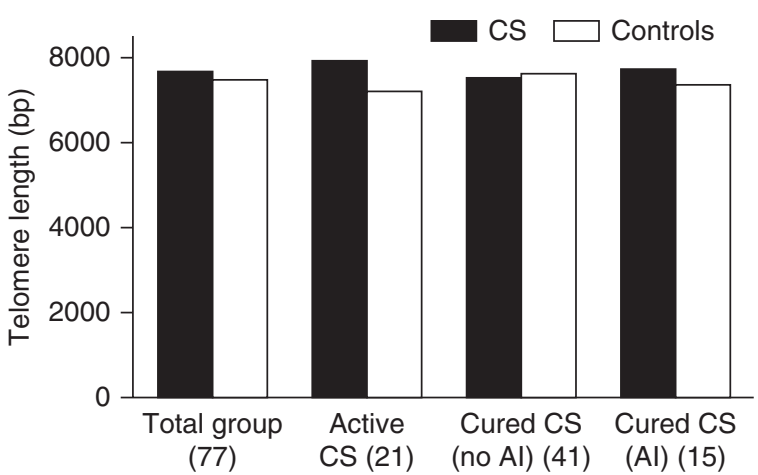

\section{Figure 1}

TL in the whole group of CS patients and controls $(7667 \pm 1260$ vs $7483 \pm 1214 \mathrm{bp})$, as well as in patients with active CS (7943 \pm 1309 vs $7230 \pm 1591$ bp), cured CS without $(7510 \pm 1219$ vs $7639 \pm 1335 \mathrm{bp}$ ) or with adrenal insufficiency (Al)

( $7727 \pm 1323$ vs $7394 \pm 1411$ bp) compared with their respective matched controls. No differences were observed. CS, Cushing's syndrome; $\mathrm{Al}$, adrenal insufficiency; $\mathrm{TL}$, telomere length.

in TL were observed related to the presence of pituitary deficiencies and/or replacement therapies either. No correlation was observed between duration of hypercortisolism and TL $(R=-0.025, P=\mathrm{NS})$, or between morning serum cortisol $(R=0.047, P=\mathrm{NS}), 24$-h urinary free cortisol $(R=0.072, P=\mathrm{NS})$ or plasma ACTH $(R=0.192, P=\mathrm{NS})$ and TL. In active CS patients, we did not observe differences in TL depending on the use of steroidogenesis inhibiting drugs (treated with metyrapone $8258 \pm 1178$ vs ketoconazole $7896 \pm 1432$, NS).

In the multiple linear regression analysis performed to identify potential predictive factors of $\mathrm{TL}$, we observed that age and dyslipidemia were negative predictive factors for TL shortening ( $P=0.006$ and $P=0.017$ respectively), while total leukocyte count was a positive predictor for TL $(P=0.043) \quad\left(R^{2}=0.23\right)$, indicating that more leukocytes were associated with longer TL. The main leukocyte cell subtypes count (neutrophils and lymphocytes) differed between active CS patients and controls (Table 2), but not between cured CS patients and their healthy controls. After adjustment for total leukocyte count as covariate, no differences in TL between the 21 active CS and their controls were observed either $(7600 \pm 1197$ vs $7450 \pm 1274, P=\mathrm{NS})$.

\section{Longitudinal analysis in CS patients evaluated both during active disease and in remission}

As expected, patients were older once remission was attained. Ten patients (66\%) clearly showed an increment of TL upon remission of CS. In five (33\%) patients, TL decreased after remission (Fig. 3), but was minimal in two and of doubtful relevance, because it was around the detection limit of $300 \mathrm{bp}$ (around 4\%) TL's variation in our population (17). Moreover, after adjustment for age as covariate, TL was shorter in active disease than after remission ( $7273 \pm 1263$ vs $7870 \pm 1039$, respectively, $P<0.05$ ) in the same patients (Fig. 3), in sharp contrast with TL shortening usually observed as age increases. No significant differences in the presence of hypertension, dyslipidemia, diabetes, or use of medications were observed between the group of patients who have increased TL during remission and those who did not have increased TL. Patients who incremented TL also decreased their BMI more after remission than those who did not increase TL ( $\left.-2.3 \mathrm{vs}-0.8 \mathrm{~kg} / \mathrm{m}^{2}\right)$, although due to the small group size, it did not reach statistical significance $(P=0.19)$. A trend for a positive correlation between TL at remission and duration of remission was also seen $(R=0.494, P=0.061)$.

\section{Discussion}

To the best of our knowledge, this is the first study to evaluate TL in this rare disease and with a relatively large series of CS patients. When investigated longitudinally, our preliminary data show that patients with active CS have a shorter TL, which become longer after hypercortisolism disappeared with effective treatment. However, in the cross-sectional case-control study comparing all patients with CS and matched controls, no differences in TL were found. This was also the case when patients with active hypercortisolism, and those considered in

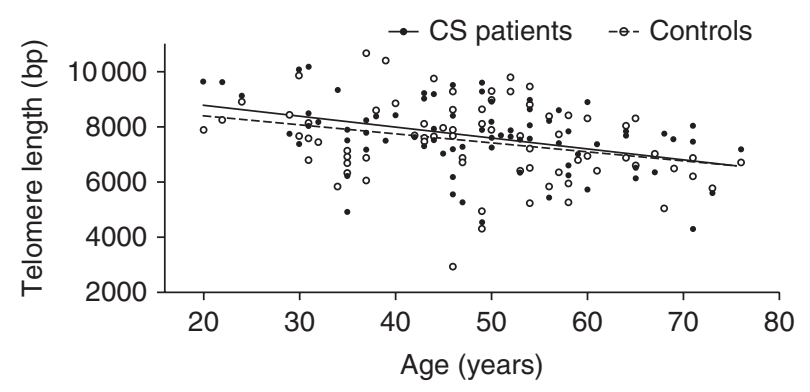

Figure 2

Telomere length in relation to age in patients with Cushing's syndrome (closed circle) and controls (open circle). Telomere length is shortened with advancing age in both $\mathrm{CS}(R=-0.400$, $P<0.001)$ and controls $(R=-0.292, P<0.01)$. 
Table 2 Total leukocyte counts and leukocyte main subsets distribution (neutrophils and lymphocytes) of Cushing's syndrome (CS) patients during active disease and remission and their matched controls. Data are expressed as mean \pm s.D.

\begin{tabular}{l}
\hline \\
\hline Leukocytes in active disease $\left(\times 10^{9} / I\right)(n=21)$ \\
Neutrophils $(\%)$ \\
Lymphocytes $(\%)$ \\
Leukocytes in cured patients without adrenal \\
insufficiency $\left(\times 10^{9} / l\right)(n=41)$ \\
Neutrophils $(\%)$ \\
Lymphocytes $(\%)$ \\
Leukocytes in cured patients with adrenal \\
insufficiency $\left(\times 10^{9} / l\right)(n=15)$ \\
Neutrophils $(\%)$ \\
Lymphocytes $(\%)$
\end{tabular}

\begin{tabular}{c}
\hline CS \\
\hline $8.8 \pm 2.3$ \\
$64.7 \pm 11.0$ \\
$24.5 \pm 9.1$ \\
$6.7 \pm 2.1$ \\
$57.1 \pm 8.2$ \\
$31.1 \pm 6.6$ \\
$6.6 \pm 1.5$ \\
$58.3 \pm 8.7$ \\
$29.6 \pm 9.6$ \\
\hline
\end{tabular}

\begin{tabular}{ccc}
\hline Controls & & $\boldsymbol{P}$ \\
\cline { 1 - 1 } $5.9 \pm 1.4$ & & $<0.01$ \\
$55.5 \pm 6.1$ & & $<0.05$ \\
$32.1 \pm 7.8$ & & $<0.05$ \\
$5.8 \pm 1.8$ & & $<0.05$ \\
$54.9 \pm 13.8$ & & NS \\
$30.9 \pm 7.1$ & & NS \\
$6.2 \pm 2.1$ & & NS \\
$52.5 \pm 7.7$ & NS \\
$34.5 \pm 6.6$ & NS
\end{tabular}

remission (with or without concomitant adrenal insufficiency), were compared with their respective matched controls.

CS patients provide a unique opportunity to examine the effects of hypercortisolism on telomere maintenance. CS determines increased morbidity and mortality, especially in the untreated state but also after therapy when compared with background population $(1,18)$. Severe morbidities are also increased even in the 3 years before diagnosis when compared with the normal population, and are not completely reversible after endocrine cure (18). The mechanisms by which CS patients do not recover completely after biochemical remission are still unknown. It is possible that telomere dysfunctions partially contribute to these abnormalities. In other situations where hypercortisolim is often present, such us chronic stress and some psychiatric conditions, TL has been found to be shorter than that in matched controls ( 6 , 9 ). These previous evidences took us to hypothesize that TL shortening could contribute to the increased morbidity and features of premature aging observed in endogenous hypercortisolism of CS. Thus, we planned this study in order to investigate the telomere system in these patients.

We have evaluated a significant number of CS patients $(n=77)$, a rare disease with an incidence ranging from 0.7 to 2.4 cases/million inhabitants per year (19). They were carefully matched for age, gender, and smoking status with controls. These relatively small groups may contribute to explain why no differences in TL were observed between CS and controls. Furthermore, many other factors apart from hypercortisolism may affect TL, both individual and environmental (genetic, epigenetic, socioeconomic status, lifestyle, growth factors, etc.) (5). In addition, TL may be affected by what is known as a 'pseudolengthening' mechanism (20); specifically, TL of lymphocytes becomes increasingly shorter than those of granulocytes over the years (17). And as a redistribution of leukocyte cell type is often seen in hypercortisolism (lymphopenia and neutrophilia), this may also affect the measured TL obtained from the total leukocyte count (21). In fact, we did find that in active disease, total leukocyte and neutrophil counts were higher and lymphocytes were lower than that observed in matched controls. We observed that total white blood cell counts in each individual blood sample also affected TL, and CS patients had higher total leukocyte counts compared with healthy controls, similar to other series (21). However, after adjustment for total leukocyte count (as a covariate), no differences in TL between CS and their healthy controls were identified.

In the multiple regression analysis, leukocytes count together with age and the presence of dyslipidemia were the predictive factors for TL, explaining $23 \%$ of the TL present in our CS patients. Not surprisingly, age was a negative predictive factor for TL, in the whole sample and in the different subgroups analysed. A positive correlation was also seen between IGF1 levels and TL, as described in healthy population $(11,22)$. Both findings support the reliability and validity of our results and the methodology used, since similar correlations have been described in much larger populations (but not in CS patients) (14); namely TL was positively correlated with serum IGF1 and negatively associated with age in a cohort of 476 healthy Caucasians aged 16-104 years (22). We also observed a negative correlation between TL and dyslipidemia as described in other paradigms, in which cholesterol has been associated with faster biological aging (23).

As expected, some baseline characteristics differed between CS and controls, such as serum morning cortisol and 24-h urinary free cortisol, certain cardiovascular risk 

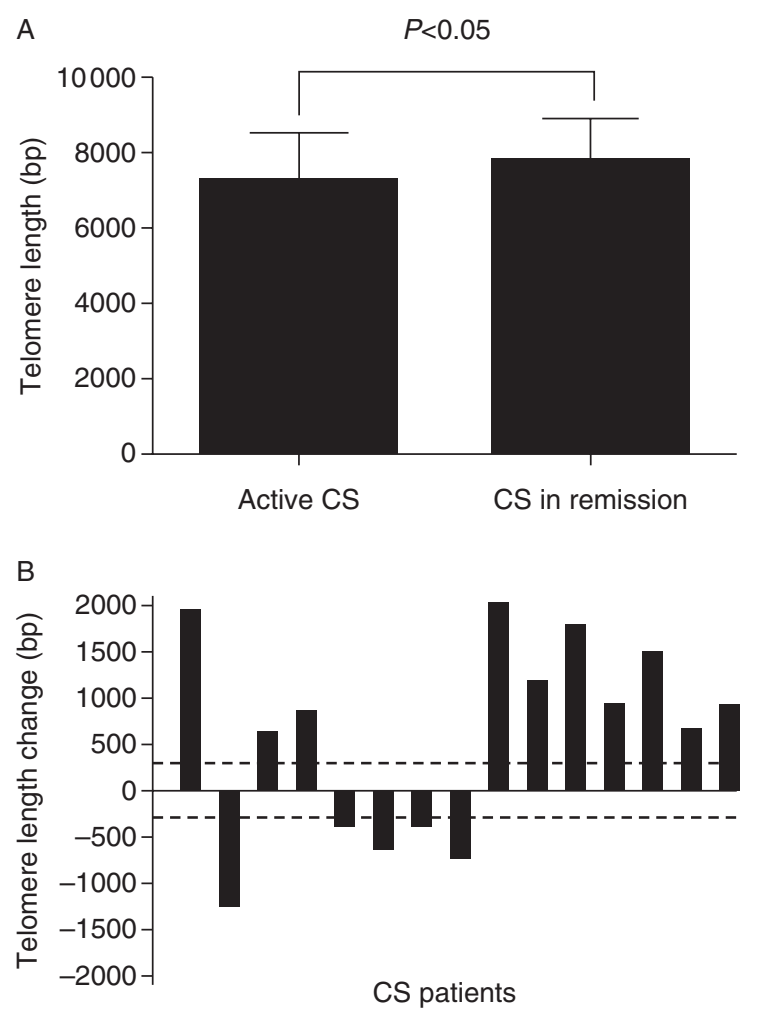

Figure 3

(A) Changes in telomere length (TL) in 15 patients in whom samples were obtained both during active hypercortisolism $(7273 \pm 1263 \mathrm{bp})$ and after remission (7870 $\pm 1039 \mathrm{bp})$. (B) TL increased in 10/15 patients, with increasing age. The dotted line shows the detection limit of the Southern blot technique.

CS, Cushing's syndrome.

factors, and psychiatric conditions (anxiety and depression), which were more prevalent in CS patients. Most of these features have recently been related to telomere dysfunctions $(9,24)$, although not all results published in the literature are concordant (25). Even though they did not seem to have impact on TL in the case-control regression analysis, with the exception of dyslipidemia which negatively affected TL, we cannot rule out that in much larger studies some of these clinical features could determine TL in some way or another. We did not find any influence of medical treatment to reduce cortisol during active disease or glucocorticoid replacement in patients with adrenal insufficiency after CS therapy on TL.

The longitudinal analysis of 15 patients evaluated both during hypercortisolism and in remission, adjusting for age (as a covariate), confirmed our initial hypothesis, because patients with hypercortisolism during active disease did have shorter telomeres than later in remission (average $596 \mathrm{bp}$ ). In spite of being $40.1 \pm 15.6$ months older at remission, TL was longer and positively associated with duration of remission. Although this finding is preliminary based on a small number of patients, and should be confirmed in the future in larger studies, it would support our initial hypothesis of a negative effect of a hyperactive hypothalamic-pituitary-adrenal axis on TL and cell senescence observed in other studies. Accelerated telomere shortening was observed in a group of 647 women (who had a sister with breast cancer) with higher perceived stress and higher levels of urinary free cortisol and cathecolamines (6). Similarly, shorter buccal cell TL was observed in children exposed to laboratory stressors with higher levels of salivary cortisol and higher autonomic reactivity (26). Greater cortisol responses and dysregulated patterns of daily cortisol secretion were associated with shorter leukocyte TL in 14 postmenopausal women caregivers of a partner with dementia compared with matched noncaregiver controls (27). Consistent with this and with our longitudinal results, one in vitro study observed how exposure to high hydrocortisone levels, comparable with those that might be reached in vivo during stress, reduced telomerase activity in lymphocytes (7). As the major pathway for telomere lengthening seems to be through telomerase activation, this could explain why a patient could have shorter TL during hypercortisolism. It is probably that when cortisol normalizes, a recovery of telomerase activity takes place, increasing TL or lowering attrition rates.

Contrary to this evidence and to our results, a recent publication showed that telomere shortening associated with hypocortisolism was observed in patients with high levels of chronic stress exposure or high degrees of inflammation, which could lead to an exhaustion of the HPA axis. It is difficult to identify the mechanism responsible for accelerated telomere shortening in hypocortisolism, often preceded by a hypercortisolemic phase in long-term chronic stress exposure, suggesting that TL could be a measure of cumulative stress (28). We found no differences in TL in our hypocortisolemic patients compared with cured patients without secondary adrenal insufficiency; an explanation could be that all adrenalinsufficient patients were correctly replaced with hydrocortisone.

Lifestyle modifications such as increased physical activity after remission may also increase TL, as reported in some studies, by inducing changes in telomerase activity. The mean fall in BMI in patients who increased TL was greater than in those who decreased TL after 
remission $\left(-2.3\right.$ vs $\left.-0.8 \mathrm{~kg} / \mathrm{m}^{2}\right)$, but did not reach statistical significance, probably due to the small sample size in the longitudinal evaluation. This change in BMI may contribute to explain the increase in TL in cured patients, similar to that seen in a recent longitudinal intervention study with Mediterranean diet, where BMI was inversely correlated with changes in TL (29).

A model of dynamic telomere balance under stress has been suggested, in which severe stress first would lead to increased turnover and depletion of circulating cells followed by a compensatory re-population when stress ends (in short stress conditions). This model could also be present in CS patients, but has to be confirmed. It would appear to be important to distinguish between true reversal of telomere shortening and replenishment by younger cells ('pseudo lengthening') that probably takes place in CS after remission (20).

The study has several limitations. The sample size, although respectable considering that CS is a rare disease, precludes any analysis in different etiological subgroups of CS. This also did not allow to control for all potential confounders, especially medical treatment during active disease, physical activity, current stress, etc. Especially in hypocortisolemic patients after surgery for CS, a perfect cortisol replacement is an elusive goal. Although the results of the longitudinal evaluation are opposite to what is expected by increasing age, and is an interesting result, it is certainly preliminary based on a small group of patients. We could not include the remaining six active patients, because four of them still had active disease and two were lost to follow-up. A larger group of patients, as well as a longer longitudinal follow-up, would clearly strengthen the conclusion of these preliminary findings. White blood cells, the most characterized tissue source for telomere studies, easily obtainable from peripheral blood, may vary in their cell type's distribution in blood as seen in CS patients. TL variability even in the same cell and for individuals of similar age complicates any conclusions on telomere biology in CS patients (30). Most studies on telomere biology and aging are much larger and crosssectional, but large scale, longitudinal, prospective, and well-designed studies are lacking. It would be interesting to evaluate TL in other tissues such as the pituitary or the adrenal in CS, because glucocorticoids induce changes in the immune system; however, this would be even more difficult than obtaining peripheral leukocytes for TL evaluation. In addition, we could not measure telomerase activity (which required fresh processing), which would provide a more direct approach to both the telomere system and its dynamics.
The main conclusion of this study is that in individual CS patients in whom hypercortisolism is controlled after successful treatment, TL increases despite being on average 3 years older. It would appear, therefore, that telomerase activity would be induced once hypercortisolism disappears, and this could be one of the mechanisms by which increased morbidity, mortality, and biological aging improve when disease is controlled. However, in the entire group of CS patients, no difference in TL was observed when compared with healthy controls, pointing to the fact that many other factors determine TL apart from age, including dyslipidemia, healthier lifestyles or differences in leukocyte subsets cell counts. Larger prospective studies are required to confirm these changes in TL in CS patients and to investigate the implications of these abnormalities further.

\section{Declaration of interest}

The authors declare that there is no conflict of interest that could be perceived as prejudicing the impartiality of the research reported.

\section{Funding}

This work was supported by grants from the Spanish Ministry of Health, ISCIII, PI 11/00001 and PI 08/0302 and by a Young Investigator Award of Fundación de la Sociedad Española de Endocrinología y Nutrición (FSEEN) to A Aulinas. J Surrallés's laboratory is funded by the Generalitat de Catalunya (SGR0489-2009) and the ICREA-Academia award. CIBERER is an initiative of the ISCIII, Spain.

\section{Acknowledgements}

The authors thank Dr Ignasi Gich for statistical advice and Dr Eulalia Urgell for advice on routine biochemical measurements.

\section{References}

1 Valassi E, Crespo I, Santos A \& Webb SM. Clinical consequences of Cushing's syndrome. Pituitary 201215 319-329. (doi:10.1007/s11102012-0394-8)

2 Pariante CM \& Miller AH. Glucocorticoid receptors in major depression: relevance to pathophysiology and treatment. Biological Psychiatry 200149 391-404. (doi:10.1016/S0006-3223(00)01088-X)

3 Aulinas A, Ramírez MJ, Barahona MJ, Mato E, Bell O, Surrallés J \& Webb SM. Telomeres and endocrine dysfunction of the adrenal and GH/IGF-1 axes. Clinical Endocrinology 201379 751-759. (doi:10.1111/ cen.12310)

4 Price LH, Kao H-T, Burgers DE, Carpenter LL \& Tyrka AR. Telomeres and early-life stress: an overview. Biological Psychiatry 201373 15-23. (doi:10.1016/j.biopsych.2012.06.025)

5 Calado RT \& Young NS. Telomere diseases. New England Journal of Medicine 2009361 2353-2365. (doi:10.1056/NEJMra0903373)

6 Parks CG, Miller DB, McCanlies EC, Cawthon RM, Andrew ME, DeRoo LA \& Sandler DP. Telomere length, current perceived stress, and 
urinary stress hormones in women. Cancer Epidemiology, Biomarkers \& Prevention 200918 551-560. (doi:10.1158/1055-9965.EPI-08-0614)

7 Choi J, Fauce SR \& Effros RB. Reduced telomerase activity in human T lymphocytes exposed to cortisol. Brain, Behavior, and Immunity 200822 600-605. (doi:10.1016/j.bbi.2007.12.004)

8 Ichiyoshi H, Kiyozuka Y, Kishimoto Y, Fukuhara S \& Tsubura A. Massive telomere loss and telomerase RNA expression in dexamethasoneinduced apoptosis in mouse thymocytes. Experimental and Molecular Pathology 200375 178-186. (doi:10.1016/S0014-4800(03)00050-9)

9 Simon NM, Smoller JW, McNamara KL, Maser RS, Zalta AK, Pollack MH, Nierenberg AA, Fava M \& Wong KK. Telomere shortening and mood disorders: preliminary support for a chronic stress model of accelerated aging. Biological Psychiatry 200660 432-435. (doi:10.1016/j.biopsych. 2006.02.004)

10 Daubenmier J, Lin J, Blackburn E, Hecht FM, Kristeller J, Maninger N, Kuwata M, Bacchetti P, Habel PJ \& Epel E. Changes in stress, eating, and metabolic factors are related to changes in telomerase activity in a randomized mindfulness intervention pilot study. Psychoneuroendocrinology 201237 917-928. (doi:10.1016/j.psyneuen.2011.10.008)

11 Müezzinler A, Zaineddin AK \& Brenner H. A systematic review of leukocyte telomere length and age in adults. Ageing Research Reviews 201312 509-519. (doi:10.1016/j.arr.2013.01.003)

12 Bayne S, Jones MEE, Li H \& Liu J-P. Potential roles for estrogen regulation of telomerase activity in aging. Annals of the New York Academy of Sciences 20071114 48-55. (doi:10.1196/annals.1396.023)

13 Babizhayev MA \& Yegorov YE. Smoking and health: association between telomere length and factors impacting on human disease, quality of life and life span in a large population-based cohort under the effect of smoking duration. Fundamental \& Clinical Pharmacology 2011 25 425-442. (doi:10.1111/j.1472-8206.2010.00866.x)

14 Sambrook J, Fritschi EF \& Maniatis T. In Molecular Cloning: A Laboratory Manual, vol 1, p 1659, 2nd edn. New York: Cold Spring Harbor Laboratory Press (ISBN 0-87969-309-6), 1989.

15 Castella M, Puerto S, Creus A, Marcos R \& Surralles J. Telomere length modulates human radiation sensitivity in vitro. Toxicology Letters 2007 172 29-36. (doi:10.1016/j.toxlet.2007.05.012)

16 Lin KW \& Yan J. The telomere length dynamic and methods of its assessment. Journal of Cellular and Molecular Medicine 20059 977-989. (doi:10.1111/j.1582-4934.2005.tb00395.x)

17 Aubert G \& Lansdorp PM. Telomeres and aging. Physiological Reviews 200888 557-579. (doi:10.1152/physrev.00026.2007)

18 Dekkers OM, Horváth-Puho E, Jorgensen JO, Cannegieter SC, Ehrenstein V, Vandenbroucke JP, Pereira AM \& Sorensen HT. Multisystem morbidity and mortality in Cushing's syndrome: a cohort study. Journal of Clinical Endocrinology and Metabolism 201398 2277-2284. (doi:10.1210/jc.2012-3582)

19 Valassi E, Santos A, Yaneva M, Tóth M, Strasburger CJ, Chanson P, Wass JA, Chabre O, Pfeifer M, Feelders RA et al. The European Registry on Cushing's syndrome: 2-year experience. Baseline demographic and clinical characteristics. European Journal of Endocrinology 2011165 383-392. (doi:10.1530/EJE-11-0272)
20 Epel E. How "reversible" is telomeric aging? Cancer Prevention Research 20125 1163-1168. (doi:10.1158/1940-6207.CAPR-12-0370)

21 Sauer J, Polack E, Wikinski S, Holsboer F, Stalla GK \& Arzt E. The glucocorticoid sensitivity of lymphocytes changes according to the activity of the hypothalamic-pituitary-adrenocortical system. Psychoneuroendocrinology 199520 269-280. (doi:10.1016/0306-4530 (94)00058-I)

22 Barbieri M, Paolisso G, Kimura M, Gardner JP, Boccardi V, Papa M, Hjelmborg JV, Christensen K, Brimacombe M, Nawrot TS et al. Higher circulating levels of IGF-1 are associated with longer leukocyte telomere length in healthy subjects. Mechanisms of Ageing and Development 2009 130 771-776. (doi:10.1016/j.mad.2009.10.002)

23 Harte AL, da Silva NF, Miller MA, Capuccio FP, Kelly A, O'Hare JP, Barnett AH, Al-Daghri NM, Al-Atlas O, Alokail M et al. Telomere length attrition, a marker of biological senescence, is inversely correlated with triglycerides and cholesterol in South Asian Males with type 2 diabetes mellitus. Experimental Diabetes Research 20122012895185. (doi:10.1155/2012/895185)

24 Fuster JJ \& Andrés V. Telomere biology and cardiovascular disease. Circulation Research 200699 1167-1180. (doi:10.1161/01.RES. $0000251281.00845 .18)$

25 Ye S, Shaffer JA, Kand MS, Harlapur M, Muntner P, Epel E, Guernsey D, Schwartz JE, Davidson KW, Kirkland S et al. Relation between leukocyte telomere length and incident coronary heart disease events (from the 1995 Canadian Nova Scotia Health Survey). American Journal of Cardiology 2013111 962-967. (doi:10.1016/j.amjcard.2012.12.017)

26 Kroenke CH, Epel E, Adler N, Bush NR, Obradovic J, Lin J, Blackburn E, Stamperdahl JL \& Boyce WT. Autonomic and adrenocortical reactivity and buccal cell telomere length in kindergarten children. Psychosomatic Medicine 201173 533-540. (doi:10.1097/PSY. Ob013e318229acfc)

27 Tomiyama AJ, O'Donovan A, Lin J, Puterman E, Lazaro A, Chan J, Dhabhar FS, Wolkowitz O, Kirschbaum C, Blackburn E et al. Does cellular aging relate to patterns of allostasis? An examination of basal and stress reactive HPA axis activity and telomere length. Physiology \& Behavior 2012106 40-45. (doi:10.1016/j.physbeh.2011.11.016)

28 Wikgren M, Maripuu M, Karlsson R, Nordfjäll K, Bergdahl J, Hultdin J, Del-Favero J, Roos G, Nilsson LG, Adolfsson R et al. Short telomeres in depression and the general population are associated with a hypocortisolemia state. Biological Psychiatry 201271 294-300. (doi:10.1016/j.biopsych.2011.09.015)

29 García-Calzón S, Gea A, Rzquin C, Corella D, Lamuela-Raventós RM, Martínez JA, Martínez-González MA, Zalba G \& Marti A. Longitudinal association of telomere length and obesity indices in an intervention study with a Mediterranean diet: the PREDIMED-NAVARRA trial. International Journal of Obesity 201438 177-182. (doi:10.1038/ijo. 2013.68)

30 Surrallés J, Hande MP, Marcos R \& Lansdorp PM. Accelerated telomere shortening in the human inactive X chromosome. American Journal of Human Genetics 199965 1617-1622. (doi:10.1086/302665)

Received 4 February 2014

Revised version received 31 March 2014

Accepted 14 April 2014 